\title{
Contribución de la Historia de las Ciencias al desarrollo profesional de docentes universitarios
}

\section{Contribution of History of Science to professional development of university teachers}

\author{
Álvaro García-Martínez \\ Facultad de Ciencias y Educación, Universidad Distrital Francisco José de Caldas, Bogotá, Colombia. \\ alvaro.garcia@udistrital.edu.co \\ Mercè Izquierdo Aymerich \\ Departament de Didàctica de la Matemàtica i de les Ciències Experimentals, \\ Universitat Autònoma de Barcelona \\ merce.izquierdo@uab.cat
}

RESUMEN • En este trabajo se analiza el papel que adquiere la Historia de la Química (HQ) en un programa de formación didáctica de profesores universitarios de química al estudiar el cambio químico, que tuvo como elemento central la réplica de instrumentos y experimentos científicos del siglo XVIII. La metodología de la investigación se inspiró en la investigación-acción, donde los docentes que participaron en el programa de formación se organizaron en una Comunidad de Desarrollo Profesional de Profesores (CODEP), que inicialmente se centró en el conocimiento erudito de la Química (desde la HQ) para irse desarrollando a una formación más compleja en didáctica de las ciencias.

PALABRAS CLAVE: Historia de las Ciencias; comunidad de desarrollo profesional de profesores; práctica experimental; instrumentos y experimentos científicos; cambio químico.

ABSTRACT - In this paper examines the role that acquires the History of Chemistry (HC) in training program chemistry teachers on didactic of science, who had as core the replica of scientific instruments and experiments of eighteenth century, when they studied the chemical change. The research methodology was inspired by action research, where teachers who participated in the training program were organized into a "Professional Development Community of Teachers (PRODEC)», which initially focused on the knowledge of chemistry (from HC) then they developed a more complex training in didactic of science.

KEYWORDS: History of Science; professional development community of teachers; experimental practice; scientific instruments and experiments; chemical change. 


\section{HISTORIA Y FILOSOFÍA DE LAS CIENCIAS EN EL CONTEXTO ESCOLAR}

El ámbito de investigación que estudia la relación entre la Didáctica de las Ciencias y la Historia y Filosofía de las Ciencias ha puesto en evidencia su importancia para el diseño de la ciencia escolar (De Hosson, 2011; Furió y Domínguez, 2007; Izquierdo, Quintanilla, Vallverdú y Merino, 2007; Níaz, 2009). Son numerosos los argumentos a favor de esta relación y destacamos algunos de ellos especialmente relevantes en nuestra investigación.

1. La Historia y Filosofía de las Ciencias proporcionan narraciones e historias que introducen a los estudiantes en la actividad científica llevada a cabo por personas «reales», con sus propias motivaciones y expectativas, que vivieron situaciones políticas y sociales concretas y que influyeron en su trabajo. También les ofrecen «modelos» con los cuales interpretan tentativamente determinados fenómenos, puesto que la discusión y el convencimiento del oponente forma parte intrínseca de esa actividad. Con ello, se ofrecen recursos importantes para una ciencia escolar en la cual aprender a dialogar es una de sus finalidades.

2. Para que un profesor de ciencias pueda ayudar a los estudiantes a entender hasta qué punto la ciencia capta el mundo real, subjetivo y vivo, ha de haberse educado en Historia y Filosofía de las Ciencias. Por ello sería extraño pensar en un buen profesor de ciencias que no tenga un conocimiento razonablemente elaborado de los términos de su propia disciplina -causa, ley, explicación, modelo, teoría, hecho- o un conocimiento de la dimensión cultural e histórica de su propia disciplina (Izquierdo, 2000). Los profesores deberían ser educados en ciencias y no simplemente ser formados en ciencias, es decir, que una educación en ciencias va más allá de «formar» en ciencias, puesto que implica una reflexión «sobre» las mismas ciencias, en otras palabras, sobre la naturaleza de la ciencia que va a enseñar.

3. Uno de los principales aportes de la Historia de la Ciencia a la formación de los profesores es hacer ver la necesidad de una aproximación fenomenológica de las representaciones científicas: los estudiantes necesitan saber con qué y cómo se relacionan dichas representaciones y poderlas así confrontar con situaciones de su vida cotidiana, o mejor aún con situaciones de la vida real en otros momentos de la evolución de la ciencia misma (García-Martínez, 2003). Haciendo uso de una analogía, se plantea que la Historia de la Ciencia se convierte en un vehículo para formar a los profesores, en formación inicial y en ejercicio, ya que no solo están estudiando su disciplina, sino que se están cuestionando la manera como se genera el conocimiento y cómo se transmite de generación en generación (Quintanilla, 2005).

Nos situamos claramente en esta línea de pensamiento y de investigación que apuesta por la vinculación de los estudios de Filosofía y de la Historia de las Ciencias (HFC) a la enseñanza de las ciencias en general. Consideramos que, con ello, se producen visiones más próximas a la manera en que se crea el conocimiento científico y a la actividad científica misma, con menos estereotipos y con una postura más crítica y reflexiva, ya que de esta manera se generan nuevas y mejores relaciones entre lo que se desea enseñar y lo que el estudiante va a aprender (punto 1). Desde nuestra perspectiva de responsables de la formación inicial y permanente de los profesores de ciencias, nuestro reto es conseguir que los profesores se eduquen en HFC (punto 2) pero de tal manera que se refleje en su práctica docente; y esto no ocurre si carecen de un conocimiento funcional para llevarla a cabo (Mellado y González, 2000). Quizá uno de los mayores obstáculos al incorporar la historia en las lecciones de química es el disponer de narrativas históricas adecuadas para ser empleadas en el contexto escolar. Y esto se refuerza con la dificultad de identificar las fuentes históricas adecuadas (el trabajo de los científicos y sus descubrimientos) que se les ofrecen y que les han de permitir enseñar mejor las ciencias, con énfasis en lo que parece ser uno de los principales escollos: el desarrollo adecuado del trabajo experimental en la ciencia escolar (punto 3) (Wandersee y Baudoin, 2002). 
La finalidad de nuestra investigación es construir una historia de la química que sea asequible a los profesores y que, a la vez, incida en los aspectos de mayor dificultad para los alumnos y que más errores generan, que son, a nuestro juicio, los que requieren una interpretación correcta de la mediación de instrumentos en el trabajo experimental. Como ejemplo, nos centramos aquí en el significado químico de lo que fueron los «fluidos» en la química del siglo XVIII: los «aires», que fueron «capturados» mediante «trampas de gases», y el "calor», que lo fue mediante el calorímetro. En ambos ejemplos se produjo una compleja construcción conceptual como resultado de esta nueva intervención experimental. Los nuevos conceptos de "sustancia gas» y "calórico» (diferenciado de calor específico y de temperatura) se explican ahora como si fueran entidades físicas evidentes y lógicas, y a los alumnos se les escapa el significado químico, tan importante para comprender de manera completa lo que es una reacción química y las leyes que la regulan (la conservación de la masa y la conservación de la energía).

Sabemos que este objetivo es difícil de lograr, pero la tarea ya ha sido abordada en varios estudios preliminares de programas de formación docente soportados en las relaciones entre Historia y Filosofía de las Ciencias que nos ofrecen un buen punto de partida (Quintanilla, 2005).

\section{La práctica experimental en la construcción del conocimiento escolar}

El estudio del progreso de la ciencia se ha abordado desde enfoques diferentes y con diversos soportes teóricos; en el momento actual son numerosas las investigaciones que resaltan el papel social y cultural de la actividad científica y de los resultados que de ella se derivan. Desde esta última postura, se resalta el papel de la práctica experimental y de los instrumentos científicos (o «artefactos» tecnológicos) en toda esta actividad de producción de conocimientos, particularmente han tenido un papel de vital importancia en el desarrollo de la química como disciplina. Esta perspectiva se ha incorporado a la Filosofía de la Ciencia (tecnografía) y a la Historia de las Ciencias, dando lugar a una línea de investigación que se ocupa del papel que desempeńaron los instrumentos científicos, cuadernos de laboratorio y los trabajos de laboratorio en el proceso de construcción de conocimientos en un momento específico de la historia de la humanidad (Holmes y Leveré, 2000; Bertomeu y García-Belmar, 2002). Desde aquí se genera un posicionamiento interesante, desde nuestra perspectiva, al estructurar un enfoque cultural para la ciencia misma, que Pickering (1992) caracteriza como el paso de la «ciencia como conocimiento» a la «ciencia como práctica y cultura». Según el mismo autor, este avance fundamental consiste en el análisis de la "práctica científica, lo que los científicos hacen de hecho, y hacia el estudio de la cultura científica, entendida como la esfera de los recursos que la práctica hace funcionar dentro y fuera de ella». La cultura implica, en palabras de Pickering (1995), las "cosas hechas» de la ciencia (habilidades, relaciones sociales, máquinas e instrumentos, así como hechos y teorías científicas).

En el contexto de la escuela, que es donde debemos incidir formando a los profesores de ciencias, la finalidad de las prácticas es contribuir a que los alumnos consigan elaborar explicaciones teóricas de los hechos del mundo y sean capaces de actuar responsablemente con los criterios científicos que son propios de la ciencia escolar. Por ello, el trabajo práctico es el núcleo central, imprescindible, de la ciencia escolar y su objetivo final es transformar lo práctico en teórico, que se sustenta en los tres pilares de la actividad científica escolar: los hechos del mundo que se han de conocer; el lenguaje y los signos en general, que deberán servir para la comunicación efectiva en los diversos niveles que se requieren: en las discusiones en clase y en los estándares que se requieren según los currículos; y los métodos (manipulaciones e instrumentos) con los que se interviene en los fenómenos (Izquierdo, Sanmartí y Espinet, 1999). La actividad científica va a transformar los «hechos» en «hechos científicos» en el marco del modelo teórico en el cual se trabaja; como ocurre con toda actividad humana, la actividad científica escolar está influenciada por ciertas variables sociales entre las cuales destacamos la construcción de explicaciones, mejor argumentadas, haciendo uso de herramientas presentes en el entorno de quien aprende (Nakhleh et al., 2002). 
Si centramos la atención en los aspectos prácticos que, como hemos dicho, constituyen la principal dificultad de la ciencia escolar, constatamos que para construir el hecho científico (para pasar del "hecho» al "hecho interpretado») se ha de saber cuál es el objeto de estudio que plantea un problema y cómo se interviene en él: modificándolo mediante instrumentos específicos y manipulando de la manera que se considera adecuada. Se requieren, por tanto, tres modelos: el modelo del fenómeno, que se refiere a la comprensión del fenómeno mismo desde lo conceptual, el modelo instrumental, que se refiere a la comprensión conceptual del instrumento y experimento, y el procedimiento material, que se ejecuta (acción manipulativa) (Pickering, 1989), los cuales contribuyen a dar sentido a las prácticas experimentales. Izquierdo et al. (1999) plantean tres preguntas para el análisis del hecho científico: ¿Qué tengo? (mediante un modelo del fenómeno), ¿Qué hago? (mediante un modelo de la acción que realiza), ¿Qué pasa y por qué pasa? (mediante un modelo del instrumento que utiliza), las cuales están relacionadas entre sí, y solamente cuando se responden las tres a la vez y de manera coherente, los datos que se obtienen tendrán sentido y la experiencia, globalmente, servirá para aprender (en filosofía, plantean las autoras, las respuestas constituyen la ontología, la metodología y la epistemología del fenómeno). Por su parte, Nakhleh et al. (2002) plantean la cognición distribuida (donde la cognición no es únicamente propiedad de la mente de los individuos, sino que puede estar distribuida también para su uso en diferentes tipos de artefactos diseńados) para estudiar el aprendizaje del trabajo de laboratorio, que se relaciona con las interacciones de las personas (aprendices y profesores) entre sí, con el medio del laboratorio y con las herramientas, tales como los instrumentos científicos empleados frecuentemente en los trabajos de laboratorio.

\section{La práctica experimental en el estudio del cambio químico desde la historia de la química}

Es bien sabido que durante la última cuarta parte del siglo XVIII y hasta la primera mitad del siglo XIX el estudio de la química se transformó debido a varios factores: la introducción de una nueva interpretación de la combustión y calcinación, un nuevo sistema de nomenclatura, su alcance de ciencia cuantitativa (debido a la balanza), la creación de modelos de conducta en el laboratorio y reglas de adecuado razonamiento experimental, la aparición de una nueva teoría de la acidez y del estado gaseoso, un nuevo concepto de sustancia simple y de composición, entre otros. La comprensión moderna de cómo se investigan los fenómenos naturales fue un producto de este periodo y la química que surgió de esta revolución fue percibida como el paradigma moderno de lo que debe ser una disciplina científica (Donovan, 1996).

Lissa Roberts (1991) plantea que para una adecuada comprensión de esta «revolución química» se ha de tener en cuenta la función de los instrumentos (artefactos y lenguajes) en el desarrollo científico. Los instrumentos, así como las teorías, nunca son pasivos. Ambos operan por la orientación de ciertas habilidades perceptuales, procesos manipulativos y organización de categorías. Normalmente no son evidentes para el ciudadano que hace uso de ellos indirectamente a través del uso de artefactos tecnológicos empleados cotidianamente. Asimismo, en las aulas, la ciencia queda lejos de los problemas y los instrumentos que generaron conceptos y teorías, y se vuelven imperceptibles para todos. Solo nos damos cuenta de su importancia cuando "parecen nuevos» (problemas e instrumentos) y quedan abiertos a la controversia, o cuando dejan de funcionar adecuadamente, perdiendo así su identidad por el uso de que se da.

Partiendo de estos referentes y centrando la mirada en el cambio químico, se observa el papel fundamental que desempeñaron los instrumentos científicos en el estudio y posterior comprensión de la combustión; entre estos destacamos la cuba neumática, la balanza y el calorímetro. 
a. La creación de la cuba neumática se debe a un proceso investigativo que culmina en un primer momento con Stephen Hales y que permitió la recolección de «vapores» con menos impurezas $y$, en consecuencia, el estudio de sus propiedades. Posteriormente, otros "filósofos naturales» después de él desarrollaron y perfeccionaron la instrumentación para el estudio de los «aires», como es el caso de Priestley. Mediante su uso no solo fue posible identificar numerosos «aires», sino también explicar cómo se generaban o cómo eran empleados como reactivos en buen número de reacciones químicas conocidas hasta entonces.

b. La balanza se había utilizado desde siglos atrás, pero es en este momento cuando se interpretan los procesos y las relaciones de masa durante el cambio químico al cuantificar todas las sustancias que entraban en un proceso (puesto que no existían sustancias sin masa, ya que los gases también la tenían). Joseph Black empleó de forma rigurosa y precisa la balanza en sus estudios sobre la disminución de masa de la «Magnesia Alba» (carbonato de magnesio) cuando se calentaba, y la interpretó como desprendimiento de un gas que estaba fijado previamente en ella. A finales del siglo XVIII la balanza pudo reemplazar al alambique como símbolo de la química porque representaba un ideal de racionalización del mundo basado en un principio de conservación (Bensaude, 2002). Así pues, la balanza es a la vez instrumento privilegiado del laboratorio de química, un concepto organizador que permite hacer abstracción de determinadas circunstancias, y un instrumento de argumentación que crea un escenario para la prueba (Bensaude y Stengers, 1997), permitiendo pasar de principios generales (que hacen evidentes ciertas propiedades) a componentes que tienen masa.

c. Finalmente, el calorímetro de hielo, creado por Lavoisier y Laplace, fue postulado por ellos para el estudio de la teoría del calor argumentando su utilidad en razón a su precisión y amplia aplicabilidad que podría permitir su adopción por otros investigadores. Para ellos, el calórico era un componente de la materia y, si bien esta afirmación fue abandonada posteriormente, su uso llamó la atención de la comunidad hacia variación del calor en el cambio químico.

El uso de la balanza, de la cuba neumática y del calorímetro permitió una comprensión más completa del cambio químico a finales del siglo XVIII.

\section{METODOLOGÍA}

Este trabajo se enmarcó en el campo de la investigación cualitativa (interpretativa) que orientó el estudio de casos de tipo longitudinal con profesores universitarios del área de Química General (tres) y Fisicoquímica (dos) que eran docentes en un programa que forma profesores de Química. Tres profesores eran químicos y otros dos, ingenieros químicos; todos con título de maestría y uno con doctorado en Inorgánica. El caso que se reporta aquí es el de la profesora Mónica, ingeniera química, con título de maestría y con 16 años de experiencia como docente de Fisicoquímica.

El colectivo de profesores trabajó bajo los presupuestos metodológicos de la investigación-acción, ya que los participantes investigaban sus propias situaciones de aula y reflexionaban sobre ellas. Se organizó un equipo de trabajo que diseñó estrategias didácticas basadas en la historia de la química, que luego de implementarlas se analizaban, a la luz de los aprendizajes generados por los estudiantes y la forma en que los mismos profesores iban mejorando su acción docente; es decir, se seguía el proceso que incluía diseño, implementación, recogida de datos, análisis, reflexión y nuevamente intervención con la acción programada por el equipo (Cullen, Akerson y Hanson, 2010).

Los docentes y el coordinador (primer autor y también profesor del programa) conformamos una Comunidad de Desarrollo Profesional de Profesores, denominada CODEP. Los profesores participaron de manera voluntaria en este programa de formación, desarrollado durante año y medio, cuyo 
objeto de estudio era el mejorar la docencia de la química en la universidad haciendo uso de la Historia y la Filosofía de la Ciencia. La CODEP se interpretó como un grupo de profesores que se reconocen como profesionales de la educación, los cuales participan en discusiones criticas y reflexivas para la toma de decisiones, en torno a su objeto de estudio, la enseñanza y el aprendizaje en su aula (e institución) y otros procesos que alli se desarrollan y que las condicionan. Esta comunidad comparte poco a poco ciertas prácticas y referentes que se van construyendo como producto de esta dinámica, que la definen y retroalimentan.

La CODEP se basó en la réplica ${ }^{1}$ de instrumentos y de experimentos científicos empleados en el siglo XVIII para el estudio de algunos fenómenos que permitirían comprender mejor la emergencia del concepto moderno de "cambio químico». Con ello se pretendía incidir en el diseño curricular que podría realizar posteriormente el profesorado y se caracterizó por las reflexiones críticas que se provocaron, promoviendo el desarrollo metacognitivo y autorregulador de los participantes y de sus alumnos (en estos últimos se hizo evidente en las entrevistas realizadas).

El proceso de formación con los profesores universitarios está organizado en cinco fases, que buscan desarrollar su papel de diseñadores de currículo: 1) identificación de propósitos y selección de contenidos, 2) selección del tópico y área de la historia de la ciencia que se va a estudiar, 3) diseño de la herramienta de enseñanza y aprendizaje, 4) implementación y análisis del proceso de aplicación de la herramienta y 5) reflexión metacognitiva sobre el proceso desarrollado y 6) rediseño curricular (los profesores generaron cambios en la estructura de las asignaturas a su cargo).

Los datos se obtuvieron mediante el uso y análisis de instrumentos para la recopilación de información, diseñados para cada una de las fases, mediante el diario del profesor (durante todo el proceso cada profesor elaboró el suyo), instrumentos de tipo metacognitivo y un registro en audio de los seminarios de la CODEP; al mismo tiempo, el coordinador de la investigación llevó su propio diario del proceso de investigación.

A los profesores se les aplicaron varios instrumentos para identificar sus ideas sobre la naturaleza de la ciencia (Naturaleza de la Ciencia, NC), sobre procesos de enseñanza y aprendizaje de las ciencias (Modelo Pedagógico Didáctico, MPD) y sobre la experimentación y conceptos relacionados con la historia de la química (fase 1). Como objeto de estudio inicial se analizaron textos de Joseph Black (1803) sobre sus trabajos con la Magnesia Alba, y de Antoine Lavoisier (1982), los relacionados con la medida del calórico, debido a que consideramos que proporcionaron nuevos factores para la comprensión del cambio químico; en estos trabajos se analizaron en detalle el papel de los instrumentos científicos, como se ha señalado: cuba neumática, balanza y calorímetro de hielo (fase 2). Los profesores asumieron su papel de aprendices y realizaron el estudio de los documentos originales de estos autores, para el caso de Lavoisier, replicaron el experimento del calorímetro de hielo, para lo cual construyeron un calorímetro partiendo de materiales que luego emplearon con sus estudiantes (fase 3). Los profesores tomaron algunos fenómenos de referencia, que fueron el centro del proceso de diseño de las unidades didácticas ${ }^{2}$ que trabajaron posteriormente con sus estudiantes, ${ }^{3}$ utilizando como instrumento el Mapa de Diseño Curricular (MDC) (fase 4) guiados de forma permanente y con el respectivo estudio de documentos sobre diseño curricular basado en unidades didácticas (fase 5).

El análisis de los datos se realizó en cuatro niveles: el preanálisis, la codificación, la categorización y la triangulación. La triangulación se caracterizó por tener una doble función: la interacción entre

1. Esta réplica material de instrumentos científicos (calorímetro de hielo) se realizó (1) estudiando en detalle el contexto histórico del instrumento y sus experimentos y del investigador que lo empleó o construyó, (2) analizando los materiales en que fue construido, (3) reconstruyendo el instrumento y (4) estudiando el uso que le dio el investigador y los experimentos realizados con este.

2. Para Química General se diseñó una unidad didáctica sobre cambio químico y estequiometria, y para Fisicoquímica una de calor específico, esta última será el centro de este documento

3. Estudiantes de primer semestre para la Química General, y de cuarto semestre para la Fisicoquímica 
personas (los propios profesores y los estudiantes) y la interacción entre instrumentos (instrumentos escritos, diarios y trascripciones de entrevistas).

\section{DISCUSIÓN DE RESULTADOS}

Los resultados se organizan según el ritmo del desarrollo del proceso de formación, es decir, siguiendo las fases de la CODEP; para ello, se presentarán centrados en el «Caso Mónica» aunque en algunos apartados aparezcan los otros profesores participantes.

\section{a) Primera fase de la CODEP}

Identificación de propósitos y selección de contenidos.

En esta fase se estudiaron las ideas sobre la enseñanza y el aprendizaje (Instrumento MPD), así como sobre la naturaleza de la ciencia (Instrumento NC, que centró la atención en la relación entre teorías y hechos, naturaleza del conocimiento científico y desarrollo del conocimiento científico). En relación con el MPD, Mónica evidenció una mezcla entre visiones tradicionales, activistas y constructivistas, aunque prima lo tradicional; en relación con la NC, mostró una mezcla entre imágenes tradicionales (positivistas) y de avanzada (en especial la pluralidad metodológica) pero de igual forma, se destaca lo tradicional.

Respecto al uso de la experimentación, Mónica la ubicó en un papel secundario, bien para establecer una relación entre teoría y práctica, corroborar hipótesis o como recurso didáctico, pero no resaltó su papel generador de conceptos y teorías en la ciencia misma ni la posibilidad de emplearla en el conocimiento escolar. En lo que hace referencia a los instrumentos científicos, ella ubicó sus argumentos en una perspectiva clásica, al considerarlos como algo necesario para el experimento, pero no mencionó la posibilidad de que estos puedan contribuir a la generación del estudio del modelo del fenómeno.

Estos aspectos, en los que Mónica se ubicó en una perspectiva tradicional, son muy importantes, ya que al analizarlos con ella, le permitió visualizar otras posibilidades de interpretación y uso, al momento de enseñar y de planear sus actividades docentes. Existe una relación entre la imagen de ciencia, incluida la experimentación, y la manera como se interpreta la enseñanza de las ciencias.

\section{b) Segunda fase de la CODEP: Selección del tópico y área de la historia de la ciencia que se va a estudiar}

En esta fase los profesores analizaron los documentos de Black y Lavoisier mencionados, estudiaron los experimentos e instrumentos empleados, replicando algunos de esos experimentos y estudiando los criterios, sobre el qué y el cómo llevarlo al aula. El análisis del proceso evidenció cómo el conocimiento se distribuye entre las personas y entre los instrumentos y experimentos desarrollados.

A partir del capítulo de Lavoisier (1982) «De los aparatos relativos a la medida del calórico» (capítulo III de la tercera parte del Tratado elemental de química), Mónica propuso el estudio del calor específico como concepto clave para la Fisicoquímica, el cual, argumenta, es difícil de aprender por parte de los estudiantes.

5 Mónica: Considero que en Fisicoquímica uno de los temas relevantes es el de calor específico, y de ahí la importancia de recopilar documentos históricos que muestren cómo surgió dicho concepto y cómo ha evolucionado. 
8 Mónica: Antes de entrar a profundizar en conceptos como calor o entalpía, se presenta bastante dificultad al explicar este tema de calor específico a los estudiantes, pues cuando se habla de calor específico a presión constante, se considera que solo es aplicable a procesos isobáricos (sistemas cerrados).

[Transcripción-Seminario: TS]

Mónica planteó que el estudio histórico del concepto calor específico (indispensable para interpretar los datos que proporciona el calorímetro) permite incidir en la evolución del concepto calor e identificó situaciones problemáticas que aparecen como eje para el estudio de los conceptos.

Después de haber escuchado las expectativas de cada uno de los participantes de la CODEP e identificar los intereses particulares, y retomando la sugerencia del concepto de calor específico, se abordaron otros documentos (Bensuade y Stengers, 1997; Brock, 1992) para ubicar a los profesores en el contexto en el cual se desarrollaron este tipo de investigaciones del siglo XVIII. En cuanto al calorímetro, ellos analizaron cómo construirlo, con qué materiales, cómo explicarles a los estudiantes su uso, y qué materiales sugerirles para que lo construyeran; decisiones que son fundamentales para la réplica de un experimento y más para la de un instrumento científico (Heering, 2005, 2006). Estas reflexiones críticas resultaron fundamentales para lograr un desarrollo profesional.

En resumen:

a. En esta fase se realizan las primeras réplicas de experimentos y se entablan diálogos como diseñadores, puesto que se trataba de comprender muy bien los fenómenos para ser llevados al aula. Los profesores seleccionaron los experimentos que creían que serían los mejores para enseñar conceptos y procedimientos en química (entre otros) por ser buenos ejemplos de esos fenómenos y porque para ellos resultó ser algo nuevo.

b. La mirada de los profesores sobre el uso de los fenómenos estudiados en su contexto de trabajo docente era diferente, puesto que estaba filtrada por los ámbitos de desarrollo profesional, personal, conocimientos y estructuras interpretativas y práctico; ${ }^{4}$ así, mientras unos veían que se podría relacionar con temas como reacciones químicas, estequiometría, el equilibrio químico, otros se cuestionaban porque no veían una relación directa con lo que enseñaban en ese momento (ley periódica, por ejemplo). Estos elementos les van permitiendo a los profesores reflexionar sobre su práctica docente, al escuchar y trabajar con otros compañeros.

c. Por interés y dinámica del grupo, se vio cómo en los primeros seminarios se dedicó mayor tiempo al estudio de los aspectos disciplinares de la química y en menor grado a la didáctica de la misma, pero esta relación se invirtió y al final la atención se centró en mayor medida en cómo llevarlo al aula y cómo hacer el seguimiento respectivo.

\section{c) Tercera fase: Diseño de la herramienta de enseñanza y de aprendizaje}

En esta fase, el trabajo en grupo por parte de los profesores giró en torno al diseño de las actividades de aula. En un comienzo se observaron algunas ideas cotidianas sobre cómo utilizar la historia de la ciencia en el aula de clase:

4. El desarrollo profesional del profesorado se asume como una serie de procesos sucesivos de autorregulación metacognitiva que llevan a un crecimiento en los ámbitos que orientan la profesión docente (ámbito personal, de conocimientos y estructuras interpretativas, y el ámbito práctico, todos ellos en el mundo personal del profesor) producto de la comprensión, puesta en práctica y de la relación entre lo que piensan, sienten y hacen en su aula y en su institución; dichos ámbitos se ven influenciados por un ámbito externo. 
135 Jorge: Pues yo lo vengo realizando desde hace algunos semestres. Principalmente lo que hago es mirar y consultar las biografías de algunos científicos que me parecen como interesantes o pertinentes. Luego las voy contando como anécdotas a los estudiantes.

[TS]

Estas ideas resaltan el papel tradicional del uso de la historia de las ciencias, en donde se ve que una primera parte del tema se presenta para motivar a los estudiantes y para que presten atención a lo que luego les explicarán, o relacionar lo que se va a estudiar con personajes reales, y que después de contarlo, se comienza el tema que se va a estudiar. Mónica no tiene claro cómo emplear en el aula la historia de la ciencia, pero se ubica en el papel de diseñadora, es decir, en la búsqueda de alternativas que le permitan llevar ese conocimiento disciplinar hacia un conocimiento para ser enseñado.

39 Mónica: He oído hablar de unidades didácticas, que personalmente siempre he tenido como esa inquietud de: ¿qué es eso?, ¿cómo se elabora?, ¿cómo seleccionar o identificar aquello que realmente sea relevante para el estudiante?, porque para un chico lo puede ser pero para otro no...

[TS]

Aquí, Mónica se planteó un interrogante básico en torno a las unidades didácticas, que será de gran utilidad, ya que estas unidades introducirán la historia de la química en el aula.

El siguiente fragmento nos muestra cómo, en el seminario, se van construyendo otras vías de utilizar la Historia de la Ciencia en el aula, en las que la comprensión de los instrumentos científicos y su funcionalidad adquiere importancia (se destacan en negrita las ideas que consideramos emergentes respecto a la relación historia-didáctica).

55 Mónica: ...La idea es recopilar información al igual que lo hemos hecho con estos documentos de química, para tener conocimiento de los autores que se ocuparon de este tema de calor específico, de si sus teorías se acoplaban o no al contexto en que se desarrollaban...

56 Coordinador: Bien, pero analicemos cómo podrían trabajar con los estudiantes una vez que ya se ha recopilado la información.

57 Mónica: Siguiendo un modelo similar de lo que usted ha realizado con nosotros, darles la documentación, se hace necesario revisar los tiempos que se requerirían para abordar esta temática en clase, debido a que el programa de Fisicoquímica es largo. Considero que antes de entrar a trabajar los conceptos que se manejan en los libros sobre calor específico, se les debería proporcionar previamente los instrumentos a los estudiantes para que los analicen, y posteriormente trabajarlos en clase [se refiere a los instrumentos científicos], pero siguiendo la misma metodología del seminario. Así al momento de realizar el trabajo de laboratorio, los estudiantes ya poseen ciertas bases para resolver situaciones como, por ejemplo, por qué se incorpora un agitador de aluminio dentro del calorímetro y por qué se debe considerar el valor de su calor específico dentro del balance total de energía del sistema.

58 Coordinador: Carlos, ¿cómo ves que se podría llevar esto al aula?

59 Carlos: ... Estoy de acuerdo con Mónica en profundizar en la cuestión de calor específico, y aún más de calor en general. ... precisamente es aquí donde se evidencian ciertas confusiones, que no son las habituales, que tratan los libros como, por ejemplo, la diferenciación entre los conceptos de calor y temperatura. Un referente histórico podría servir de punto de partida para la comprensión de dichos conceptos en comparación con lo que se trabaja actualmente.

60 Pedro: ... Seleccionamos algunos experimentos sencillos y descartamos los de mayor complejidad. Con Carlos pensamos en elaborar una lectura en la que se describieran estos experimentos, con el fin de que ellos la analizaran y luego plantearan las posibles reacciones químicas...

[TS] 
Los profesores van concretando poco a poco sus propuestas de cómo hacer uso de la historia de la ciencia para mejorar la enseńanza y el aprendizaje. Retomando estas ideas se decidió diseñar una unidad didáctica partiendo de las propuestas de iniciar con el estudio del calorímetro de hielo de Lavoisier y haciendo algunos experimentos propuestos por este, haciendo un contraste con los métodos actuales de calorimetría. El coordinador desarrolló una serie de seminarios para orientar la construcción de unidades didácticas. En estos espacios, cada uno de los docentes escribía de manera individual sus aportes sobre las diferentes partes de la unidad didáctica y luego se presentaban al grupo de trabajo; allí se escuchaban y analizaban, complementándose o reformulándose, dependiendo de los acuerdos del grupo, para crear de esta manera una versión unificada. Así, se diseñaron los objetivos, la evaluación, la estructura, las metodologías de trabajo en el aula y los contenidos. Para la selección de los contenidos se elaboraron Mapas de Diseño Curricular (MDC), ${ }^{5}$ que permitieron a los profesores y al coordinador llegar a acuerdos sobre lo que se iba a enseñar y la secuencia. De manera coherente con la dinámica de trabajo en la CODEP, las actividades de aula se plantearon como problemas abiertos, y las de laboratorio como problemas abiertos de tipo experimental.

Los resultados en esta fase pueden resumirse de la manera siguiente:

a. En esta parte se evidenció la experticia de Mónica, que se fundamenta en su experiencia de trabajo de aula y en su formación académica, lo cual le permitió programar y actuar en consecuencia.

b. Los trabajos experimentales se orientaron con mucha naturalidad mediante el diagrama heurístico de Gowin, y las actividades fueron de introducción de conceptos, estructuración, generalización y aplicación (Sanmartí, 2002).

c. La evaluación fue en realidad una metarreflexión sobre lo que se había ido trabajando y, por lo tanto, se sitúa ya en el campo de conocimiento de la DC. ${ }^{6}$ Se planteó la idea de hacer mapas conceptuales para contrastar el aprendizaje de los estudiantes, realización de un test de ideas previas, realizar una autoevaluación y una evaluación final.

\section{d) Cuarta fase: Implementación de la unidad didáctica diseñada}

Se hace evidente la forma en que Mónica se apropió de lo diseñado y de la forma de desarrollarlo. Emergió un interés por las ideas de los estudiantes que no se había manifestado anteriormente. En el siguiente fragmento de su diario se ve cómo ella hace el análisis de lo que se ha trabajado con los estu-

5. El Mapa de Diseño Curricular, MDC, es una herramienta de diseño curricular a nivel conceptual, de orientación de las actividades docentes y de la evaluación del trabajo de aula, la cual ha sido concebida en el desarrollo de este proyecto de investigación. Consiste en un mapa que se construye a partir de la selección de los conceptos que se van a enseñar, los cuales se organizan de acuerdo con el nivel de complejidad y abstracción para ser enseńados y aprendidos. En este sentido, los conceptos más concretos y simples se ubican en la parte inferior del mapa y los más abstractos y complejos se van ubicando hacia arriba, de tal manera que el concepto clave que va a ser enseñado (de mayor complejidad y abstracción) se ubicará en la parte superior del mapa. El MDC permite que los profesores se pongan de acuerdo en los niveles de complejidad de los conceptos que enseñar, lo cual permite a su vez determinar su secuenciación a la hora de ser desarrollados, para lo cual van creando franjas que separan niveles de conceptos en el mapa. Los criterios para construirlo se soportan en la (1) selección de los conceptos básicos desde el área de conocimiento; (2) organización de estos por niveles de complejidad, lo cual está determinado por el área de conocimiento, y (3) demanda cognitiva de los conceptos, a la luz de la experiencia del profesor cuando lo ha enseñado (proceso de transposición didáctica). Este proceso de construcción conjunta del MDC desarrolla la reflexión crítica en el profesorado, que influye en sus ámbitos personales, de sus conocimientos y estructuras interpretativas y su práctica, posibilita su autorregulación y promueve el aprendizaje en la CODEP.

6. Consideramos que la Didáctica de las Ciencias es una metadisciplina en cuanto que es una manera de mirar el conocimiento científico dando prioridad a todo aquello que tiene que ver con su emergencia en contextos de enseńanza. 
diantes, lo que se va a trabajar y la forma de llevarlo a cabo, en donde resalta el papel del instrumento, del procedimiento experimental y la comprensión conceptual:

Mónica: Antes de iniciar la aplicación de las actividades 1 y 2, se aclara a todo el grupo la metodología que se va a seguir en esta unidad didáctica, ya que lo que se busca es la construcción de un concepto (calor específico). Por esto, se menciona que se partirá de la comprensión conceptual del fenómeno, se continuará con el trabajo práctico experimental y se finalizará con la interpretación del concepto...

[Diario de la profesora, DP]

Ella también escribe algunas ideas erradas que tienen los estudiantes, que luego retomará para trabajarlas en clase a la luz de otras actividades planeadas o propuestas por ella para tal fin (regulación).

... Nota: Algunos estudiantes afirmaron lo siguiente (lo cual es incorrecto). El flogisto es: Cantidad de energía que tiene un cuerpo, - Energía liberada por la sustancia cuando ocurre combustión.

$[\mathrm{DP}]$

Mónica reflejó claridad frente a lo que se planeó y la forma de hacerlo: partir de un fenómeno concreto contextualizado históricamente y, haciendo uso del instrumento, mejorar la comprensión de los estudiantes del fenómeno y de los conceptos implicados en este. Ella resaltó las dificultades presentadas por los estudiantes, al tener que enfrentarse a una metodología diferente y ante una forma distinta de abordar el estudio de un fenómeno y sus conceptos, es decir, llegar al modelo del fenómeno a partir del estudio del instrumento y la intervención experimental en el fenómeno, desde un problema histórico.

Vimos cómo ella comenzó a argumentar sobre el papel del instrumento científico para poder desarrollar la experimentación en el aula, contextualizado a la Fisicoquímica; en la entrevista menciona:

52 Mónica: ...es más impactante para ellos, haberse puesto en la tarea de diseñar sus propios calorímetros en la tarea de haber mirado los materiales, de haber dicho yo no quiero la manguera aquí en esta posición sino la quiero acá, en haberse puesto a pensar qué le voy a colocar en la parte del metal, algunos trajeron..., coladeras metálicas..., es decir, el enfrentarse ellos a esa problemática de tratar de mirar ese calor específico, cómo es y entender qué es, esto hace que para ellos, sea más impactante que manejarlo solo teóricamente...

[Entrevista a profesores]

En resumen, vimos que la postura innovadora que adquirió Mónica le fue proporcionando el liderazgo del grupo. Esta se pone en evidencia:

a. al realizar una práctica adicional para contrastar algunas variables con el calorímetro de Lavoisier y el calorímetro actual, no conformándose solo con desarrollar el trabajo propuesto, sino creando nuevas variantes para mejorarlo,

b. al incorporar las sustentaciones orales argumentadas a los estudiantes, una vez se finalizó el trabajo de laboratorio, con el fin de realizar una mejor evaluación de sus aprendizajes,

c. cuando ella usó la historia de la ciencia para que los estudiantes fueran identificando variables, les fue creando problemas que implicaban la construcción de explicaciones y de argumentos y la necesidad de decidir, como en el caso del calórico. 


\section{e) Quinta fase: Análisis del proceso de aplicación y reflexión metacognitiva sobre lo desarro- llado}

En esta fase los profesores realizaron un análisis metacognitivo sobre el proceso, reflexionaron sobre su actuación y sobre las argumentaciones generadas por los estudiantes, así como en sus aprendizajes generados. Los profesores coinciden en el choque que tuvieron los estudiantes con la metodología, las dificultades que presentaron estos para tomar el ritmo, la cantidad de esfuerzo que demanda esta, y el mayor tiempo que exige para su desarrollo. También resaltaron sus bondades frente a la mejor comprensión que generó en los estudiantes y el aporte revertido hacia ellos mismos en su enseńanza y en su desarrollo profesional.

Durante la entrevista realizada a los estudiantes sobre el proceso desarrollado, destacaron el aporte que genera el conocer las interpretaciones históricas y contrastarlas con la actual. Mencionan que el proceso de construcción del instrumento científico les permitió dilucidar interrogantes que tenían, así como generar nuevos problemas, los cuales pueden ser abordados por ellos de manera autónoma o con la asesoría del profesor, lo cual genera una dinámica de verdadera actividad científica escolar.

Frente a la intervención de Mónica, un estudiante manifestó lo siguiente:

62 Juan Carlos: ...me parece interesante que al principio para abordar el tema ella aplicó un mapa conceptual, para ver los conocimientos previos que tenía cada persona y cómo lo relacionábamos, y de acuerdo a eso ella hacía alusión al tema mediante las lecturas de la actividad de Lavoisier, y después la socialización, que era donde cada grupo exponía lo que había entendido de la lectura y ella iba aclarando las dudas, e iba como complementando todo lo de los grupos y después se sacaba como una conclusión general, que ya era como la generalización del tema como en clase..... finalmente, vino la sustentación, a través de la cual ella comprueba si uno aprendió realmente o no, entonces ella establece relaciones con los temas trabajados en clase, es un proceso bueno.

[Entrevista a estudiantes]

Tomando como referencia lo planteado por varios estudiantes, se le pidió a Mónica que reflexionara sobre cómo consideraba que ha influido esta metodología en la imagen que tenían los estudiantes de ella. Ante esto, manifestó que esta se ha fortalecido, en sentido positivo: primero por la diversidad de actividades desarrolladas, alejadas de una visión tradicional, también por la modalidad de preinforme e informe (realizado mediante diagramas UVE), por las sustentaciones que se realizan al finalizar los temas, por la interacción que se ha desarrollado con ellos, el hacer discusiones y socializaciones frente al estudio de problemas; todo esto provoca que la imagen se fortalezca puesto que sus prácticas docentes se han enriquecido.

En esta fase se hizo evidente cómo los profesores manifestaron que una de las primeras situaciones, que influye fuertemente en el proceso de implementación, es la tradición que tienen los estudiantes que se han formado en un modelo clásico, en el que el profesor es el centro del proceso docente y ellos asumen un papel pasivo, lo cual generó cierto rechazo al comienzo del proceso. El uso de la historia se hace más importante para los profesores al reconocer la manera en que esta influye en su enseñanza y en la forma como los estudiantes van mejorando su aprendizaje cuando hacen uso de los instrumentos científicos. Permite, por ejemplo, que se aborden de modo diferente los trabajos prácticos puesto que al diseñar sus propias hipótesis e instrumentos ponen a prueba sus modelos y argumentan sus hallazgos, deben relacionar cada vez mejor el pensar, actuar y hablar como fundamento de la actividad científica escolar. En esta fase los profesores realizaron una reorganización de sus asignaturas, diseñando cuatro unidades didácticas para cada curso, siguiendo la metodología implementada en la CODEP. 
En esta fase podemos sintetizar los resultados de la siguiente manera. En relación con las ideas de Mónica antes y después del trabajo en la CODEP, se evidenció un fuerte progreso:

a. en su distanciamiento de los postulados tradicionales, en lo referente a la metodología, la evaluación y los contenidos, mientras que en los objetivos y en el papel del profesor aún persisten ciertas ideas tradicionales. Lo que sí está claro es que las ideas iniciales se han modificado, dirigiéndose poco a poco hacia perspectivas alternas, distanciadas del tradicional y el activista tecnológico;

b. en la forma de interpretar el uso de la historia de la química en su trabajo docente;

c. en la manera como diseñó y planificó sus actividades de aula, y cómo las desarrolló;

$d$. en las modificaciones que realizó a la luz de los resultados que se van generando en su aplicación, $\mathrm{y}$

e. en cómo se puede hacer mejor el seguimiento de los aprendizajes de los estudiantes.

\section{CONCLUSIONES}

Esta investigación nos permite proponer algunas conclusiones.

1. Tal como se preveía, los diálogos, las reflexiones, las réplicas y los diseños generados soportados en el estudio de la historia de la ciencia y la réplica de experimentos e instrumentos científicos situaron a los profesores como aprendices, lo que les permitió desarrollar nuevas posibilidades de actuación docente como diseñadores de clases innovadoras. Este último rol se hizo evidente, por ejemplo, en los procesos de réplica mediante los cuales construyeron los instrumentos para sus clases, lo cual se constituyó en potentes actividades generadoras de actividades científicas escolares. El conocimiento de la historia de la ciencia ha brindado un soporte a la hora de programar y adelantar el trabajo de aula, dando mayor seguridad para desarrollar las actividades, para modificarlas y para generar otras propuestas nuevas.

2. Al analizar el proceso desarrollado con los profesores universitarios se observó que la apropiación del rol de aprendiz ha permitido desarrollar un proceso de formación docente que favorece el surgimiento de una Comunidad de Desarrollo Profesional Docente. Se puede considerar que la duda introducida al adoptar el papel de aprendiz ha hecho posible la apertura a nuevas perspectivas de incorporación de conocimientos didácticos y con ello se generó un espacio de reflexión que constituyó esta CODEP.

3. Por ello, a medida que va evolucionando la CODEP, el interés y el grado de importancia se va desplazando del conocimiento centrado en el conocimiento erudito de la química hacia la didáctica de la química, dándose un primer paso hacia el desarrollo profesional docente. De esta manera se ve cómo el enfoque cultural, que proporciona la historia de la química, desencadena el interés por la didáctica de las ciencias. El interés que suscita en los profesores el análisis y las reflexiones sobre la forma como se han generado los conocimientos en su disciplina les ayuda a mejorar la comprensión de lo que enseñan a sus estudiantes. Una vez se han llevado al aula las diferentes propuestas diseñadas, la comunidad desempeńa un papel fundamental a la hora de generar un espacio para la reflexión, crítica constructiva y propositiva, potenciando diferentes tipos de procesos metacognitivos en los profesores, fundamentales en el desarrollo profesional docente.

4. En este proceso de desarrollo de la CODEP, del tránsito de la disciplina hacia la didáctica, surgieron diversos roles que dinamizaron a la comunidad. Aparecieron líderes como Mónica, por ejemplo, que orientó más la argumentación teórica y el análisis de los procesos; otros que guiaron y desarrollaron el trabajo experimental y la creación de prototipos, y otros que fueron gene- 
rando reflexiones continuas sobre el cómo relacionar lo estudiado en el seminario con el trabajo de aula de los estudiantes. Estos últimos son los que poco a poco van haciendo reflexionar a sus compañeros para que se vaya desplazando la atención de la ciencia erudita hacia la ciencia escolar. Estos líderes son fundamentales porque orientan, halan e impulsan las comunidades a través de su unión y focalización de intenciones, pero su ausencia frena y disipa los esfuerzos de la comunidad. Estos tipos de roles van ligados a los ámbitos que orientan la profesión docente, es decir, los que la soportan y la proyectan, de tal modo que van ligados a aspectos personales de la práctica docente fundamentada en la interpretación de la enseñanza como actividad intelectual y práctica a la vez y, finalmente, todo esto condicionado por la influencia externa.

5. Los profesores fueron generando expectativas, intereses, ritmos y aprendizajes diferentes, lo cual influyó en la manera como cada uno de ellos va desempeńándose en la comunidad. El grado de apropiación de la metodología no es el mismo y los cambios que presentaron no son de la misma forma, ni de la misma naturaleza, inclusive no se da en todos los aspectos en todos los profesores. A pesar de los niveles de apropiación de la metodología dados por los profesores, aún existen ideas tradicionales en algunos aspectos, lo cual nos lleva a plantear que pueden generarse modificaciones en la práctica docente alejadas de posturas tradicionales pero algunas ideas "tradicionales» todavía se mantienen. El desarrollo profesional docente puede darse en un solo ámbito de formación, no requiere que se dé en todos, es un proceso lento que a veces presenta avances y retrocesos como todo proceso humano y que depende de la persona; en unos pueden ser mayores los cambios que en otros. No es continuo ni lineal, no se avanza en todo a la vez ni al mismo ritmo, todo va filtrado por los ámbitos de formación. Lo anterior se vio en los resultados de los instrumentos NC y MPD, en donde se evidenció una clara tendencia al distanciamiento que toman los profesores sobre el modelo tradicional tras haber trabajado en la Comunidad, pero persiste un eclecticismo marcado, mezclando visiones entre lo tradicional y lo avanzado, desde la naturaleza de la ciencia, y lo tradicional y alternativo, desde los modelos pedagógicos.

6. Finalmente, es importante mencionar que como producto del trabajo en la comunidad, se implementaron nuevas unidades didácticas que generaron nuevas dinámicas de aprendizaje, debido a que se desarrollaron en torno a problemas y se generó un sistema de evaluación de forma procesual. Los profesores rediseńaron los contenidos de sus asignaturas y la forma de presentarlos. Se desarrollaron seminarios cuyas temáticas fueron temas propios de la didáctica de las ciencias, se diseñaron instrumentos de ideas previas, mapas conceptuales, diagramas heurísticos, se realizó todo un proceso de formación soportado en el diseño curricular que potenció el desarrollo de la CODEP.

Es importante hacer notar que todo lo alcanzado no se logró únicamente por introducción de la historia de la química, pero su papel fue muy importante, tanto por su impacto en las ideas de los profesores como por colocar en evidencia la importancia de los instrumentos en la emergencia de conceptos, y el estudio de fenómenos y los conceptos implicados en este, lo que habitualmente no forma parte de la docencia tradicional de la química. Se puede considerar que un determinado hecho histórico se ha convertido en un hecho didáctico y que hemos podido aclarar un poco este proceso de transformación. 


\section{REFERENCIAS BIBLIOGRÁFICAS}

Bensaude V. y Stengers, I. (1997). Historia de la química. Madrid: Addison - Wesley.

Bensaude, V. (2002). La balanza: ¿Un instrumento revolucionario? En: A. Bertomeu y García (eds.). Abriendo las cajas negras: Los instrumentos cientificos de la Universidad de Valencia. Valencia: Universitat de València.

Bertomeu J. y García-Belmar, A. (eds.) (2002). Abriendo las cajas negras: Los instrumentos científicos de la Universidad de Valencia. Valencia: Universitat de València.

BLACK, J. (1803). Lectures on the Elements of chemistry. Edinburg: ed. John Robinson. Vol. 1.

Brock, W. (1992). Historia de la Química. Madrid: Alianza Editorial.

Cullen, T.; Akerson, V. y Hanson, D. (2010). Using Action Research to Engage K-6 Teachers in Nature of Science Inquiry as Professional Development. Journal of Science Teacher Education, 21. http://dx.doi.org/10.1007/s10972-010-9218-8

De Hosson, C. (2011). Una controversia histórica al servicio de una situación de aprendizaje: Una reconstrucción didáctica basada en diálogo sobre los dos máximos sistemas del mundo de Galileo. Enseñanza de las ciencias, 29(1), pp. 115-126.

Donovan, A. (1996). The chemical revolution and the modern image of science. En: M. Izquierdo (coord.). Lavoisier i els orígenes de la química modern, 200 anys després (1794- 1994). Barcelona: Societat Catalana d'Història de la Ciencia i de la Técnica.

Furió, C. y Domínguez, M. (2007). Problemas históricos y dificultades de los estudiantes en la conceptualización de sustancia y compuesto químico. Enseñanza de las ciencias, 25(2).

García-Martínez, A. (2003). Aportes del estudio histórico de instrumentos científicos a la formación del profesorado de ciencias. Tesis de Maestría, Universidad Autónoma de Barcelona, España.

HeEring, P. (2005). Weighing the Heat: The Replication of the Experiments with the Ice-calorimeter of Lavoisier and Laplace. En: Marco Beretta (ed.). Lavoisier in Perspective. Deutsches Museum, pp. $27-42$

Heering, P. (2006). Public Experiments and their Analysis with the Replication Method. Science \& Education, 15. http://dx.doi.org/10.1007/s11191-006-9013-2

Holmes, F. L. y Levere, T. H. (2000). Instruments and Experimentation in the History of Chemistry. MIT Press: Cambridge MA.

IzQuierdo, A. M. (2000). Fundamentos epistemológicos de la didáctica de las ciencias. En: F. Perales y P. Cañal (eds.). Didáctica de las ciencias experimentales. Teoría y práctica de la enseñanza de las ciencias. Alcoy: Marfil.

IzQuierdo, M.; Sanmartí, N. y Espinet, M. (1999). Fundamentación y diseño de las prácticas escolares de ciencias experimentales. Enseñanza de las Ciencias, 17(1), pp. 45-59.

IzQuierdo, Quintanilla, Vallverdú y Merino (2007). Una nueva reflexión sobre la historia y filosofía de las ciencias y la enseñanza de las ciencias. En: Historia de la ciencia. Aportes para la formación del profesorado. Santiago de Chile: Arrayán Editores.

Lavoisier (1982). Tratado elemental de química. Traducción de Ramón Gago Bohórquez. Madrid: Alfaguara, S.A.

Mellado, V. y González, T. (2000). La formación inicial del profesorado de ciencias. En: F. Perales y P. Cañal (eds.). Didáctica de las Ciencias Experimentales. Alcoy: Marfil.

Nakhleh, Polles y Malina (2002). Learning Chemistry in a Laboratory environment. En: Chemical Education: Towards research-based practice, pp. 69-94. Netherlands: Kluwer academia publishers. 
NíAz, M. (2009). Progressive transitions in chemistry teachers' understanding of nature of science based on historical controversies. Science \& Education, 18. http://dx.doi.org/10.1007/s11191-007-9082-x

Pickering, A. (1989). Living in the material world. En: Gooding, Pinch y Schaffer (eds.). The uses of experiment. Cambridge: The Cambridge University Press.

Pickering, A. (1992). From Science as Knowledge to Science as Practice. En: A. Pickering (ed.). Science as Practice and Culture. Chicago: The University of Chicago Press.

Pickering, A. (1995). The Mangle of Practice: Time, Agency \& Science. Chicago: The University of Chicago Press. http://dx.doi.org/10.7208/chicago/9780226668253.001.0001

Quintanilla, M. (2005). Historia de la ciencia y formación del profesorado: Una necesidad irreductible. Segundo congreso sobre Formación de Profesores de Ciencias. Bogotá: Revista de la Facultad de Ciencias y Tecnología, número extra.

RoberTs, L. (1991). A word and the world. The significance of naming the calorimeter. ISIS, 82, pp. 198-222 http://dx.doi.org/10.1086/355725

Sanmarti, N. (2002). Didáctica de las ciencias en la educación secundaria obligatoria. Madrid: Síntesis Educación.

Wandersee, J. H. y Baudoin, P. (2002). The History of Chemistry: Potential and Actual Contributions to Chemical Education. En: J. K. Gilbert, O. De Jong, R. Justi, D. F. Treagust y J. H. Van Driel (eds.). Chemical Education: Towards Research-based Practice. Kluwer Academic Publishers, Dordrecht. 


\title{
Contribution of History of Science toward professional development of university teachers
}

\author{
Álvaro García-Martínez \\ Facultad de Ciencias y Educación, Universidad Distrital Francisco José de Caldas, Bogotá, Colombia. \\ alvaro.garcia@udistrital.edu.co \\ Mercè Izquierdo Aymerich \\ Departament de Didàctica de la Matemàtica i de les Ciències Experimentals, \\ Universitat Autònoma de Barcelona \\ merce.izquierdo@uab.cat
}

The field of research that studies the relationship between Didactic of Science and History and Philosophy of Science has demonstrated its importance for the design of school science. In this context, we clearly find ourselves in this line of thought and research which is committed to linking the study of History and Philosophy of Science (HPS) to the teaching of Science in general. We believe that with this vision people can generate images that are closer to the way that scientific knowledge and scientific activity itself is created, with fewer stereotypes and more critical and reflective stance. Likewise, new and better relationships are generated between what you want to teach and what students will learn.

From our perspective, as responsible for the initial and continuing training of Science teachers, our challenge is to ensure that teachers be educated in HPS but in a way that is reflected in their daily teaching. We have shown that one of the biggest obstacles to incorporate History lessons in Chemistry is the availability of adequate historical narratives to be used in the school context. And this is reinforced by the difficulty of identifying appropriate historical sources (the work of scientists and their discoveries in this case) to be offered and that should allow them to better teach science, with emphasis on what appears to be one of the main obstacles: the proper development of the experimental work in school science.

The purpose of our research was to build a History of Chemistry affordable to teachers and, in turn, which highlighted the most difficult aspects for students because, in our view, they required the proper interpretation of the mediating instruments in the experimental work. Thus, in this study we analized the role the History of Chemistry (HC) acquired in a program designed to train university Chemistry teachers, which had as its central element "the replication of scientific experiments and instruments" of the eighteenth century, while they also studied chemical change.

The research methodology was qualitative type (interpretative) and it was oriented to a study case of longitudinal type with Chemistry university professors (five teachers participated, but for this document just the Monica case is reported). They participated in the training program and they organized in a "Professional Development Community of Teachers (PRODEC)" in order to improve their teaching of Chemistry. This program initially focused on knowledge of Chemistry (from HC) and then developed into a more complex training in didactic of Science.

The PRODEC developed the next steps in the design process of scientific school activities: 1) identification of purpose and content selection, 2) selection of topic and area of History of Science to be studied, 3) design of teaching and learning tools, 4) application and analysis of the implementation process of the tool, 5) metacognitive reflection about the process developed and 6) curriculum redesign (the teachers generated changes in the structure of the subjects under their responsibility).

Finally, as we expected, the dialogues, reflections, replicas and generated designs, supported in the study of the History of Science and replication of experiments and scientific instruments placed teachers as learners, which allowed them to develop new possibilities of teaching performance as designers of innovative lessons. The knowledge of the History of Science has given support in scheduling and advances in classroom work, providing greater security in the activities and approaches to modify and generate new proposals. 